https://doi.org/10.24201/aap.2021.319

ARTÍCULO

\title{
Seguridad humana. Ciudadanía y Estado-nación en India
}

\section{Human Security. Citizenship and Nation-state in India}

\author{
LAURA CARBALLIDO CORIA \\ https://orcid.org/0000-0002-2447-6746
}

Universidad Autónoma Metropolitana (Cuajimalpa), México

Recepción: 1 de octubre de 2020

Aceptación: 4 de enero de 2021

Resumen: En este texto se examina un par de acciones que revisten particular gravedad, pues son ejercidas y apoyadas por el aparato estatal y ponen en duda los principios bajo las cuales se construyó la democracia en India. La primera es la decisión de hacer una enmienda a la Ley de Ciudadanía contenida en la Constitución, y la segunda es la actualización del Registro Nacional de Ciudadanos, cuya primera fase se ha llevado a cabo en Assam. Ambas confirman la visión hindú que de la nación y de la ciudadanía tiene en el gobierno actual y colocan en una situación vulnerable a la comunidad musulmana en particular, aunque no únicamente.

Palabras clave: ciudadanía; Estado-nación; migración; lengua; Partición.

\begin{abstract}
This text analyzes a pair of actions that have serious consequences, since they are executed and supported by the state. They endanger the principles under which democracy was built in India. The first one is the amendment to the Law of Citizenship in the Constitution and the second is the update of the National Register of Citizens that has been implemented in Assam. Both confirm the way the current government sees nation and
\end{abstract}


citizenship as predominantly Hindu. Also, these mechanisms render the Muslim community and others vulnerable.

Key words: citizenship; Nation-state; migration; language; Partition.

Hablar de seguridad humana es hablar de la posibilidad de una vida estable, libre de amenazas. Éstas pueden ser de orden diverso: deterioro ambiental, crisis económica, políticas estatales que vulneran a sus poblaciones, por ejemplo, en detrimento de la equidad de género, y conflictos religiosos (Commission on Human Security, United Nations 2003). ${ }^{1}$

En India independiente hemos visto el uso de la religión por parte de los gobiernos central y estatales y de los partidos políticos. Reviste especial importancia el ascenso de los grupos hindúes chauvinistas, ejemplificados en el partido político Bharatiya Janata Party (BJP de aquí en adelante) y en organizaciones, como la religiosa Bajrang Dal, la estudiantil Akhil Bharatiya Vidyarthy Parishad y la de trabajadores Bharatiya Kishan Sangh.

El fortalecimiento de estos grupos a partir de la década de 1960 se ha visto acompañado de iniciativas que fomentan el odio hacia musulmanes y cristianos principalmente, pero también hacia grupos que ellos ubican en los márgenes del hinduismo. En muchas ocasiones estas iniciativas se han traducido en actos de violencia, como ataques a iglesias y a personas sospechosas de haber matado o comido carne de res ("India probe after 'cow vigilantes kill Muslim man'” 2017).

Estas acciones han adquirido un tono distinto desde que el BJP tomó el poder en 19982004 y, posteriormente, en dos periodos consecutivos, 2014-2019 y 2019 a la fecha. Esto se debe a que diversas medidas impulsadas por el gobierno han minado seriamente la seguridad humana en el país.

En este texto abordaré un par de estas acciones que revisten particular gravedad, pues son ejercidas y apoyadas por el aparato estatal y ponen en duda los principios bajo las cuales

\footnotetext{
${ }^{1}$ Agradezco los comentarios y sugerencias de los dos dictámenes anónimos: fueron relevantes y me permitieron afinar varias ideas a lo largo del artículo.
} 
se construyó la democracia india, contenidos en la Constitución. La primera es la decisión de hacer una enmienda a la Ley de Ciudadanía y la segunda es la actualización del Registro Nacional de Ciudadanos, cuya primera fase se ha llevado a cabo en Assam. Ambas confirman que el gobierno actual posee una visión excluyente, basada en lo hindú, de la nación y la ciudadanía, y colocan en una situación vulnerable a la comunidad musulmana en particular, aunque no únicamente, como veremos más adelante. Cabe aclarar que otras decisiones y acciones del gobierno indio resultan también preocupantes, pero, por cuestiones de espacio, he decidido centrar mi análisis en estas dos. ${ }^{2}$

La enmienda a la Ley de Ciudadanía permite que extranjeros que hayan entrado a India sin la documentación debida puedan solicitar la ciudadanía. La enmienda contempla a los extranjeros que pertenezcan a las comunidades hindú, sikh, budista, jaina, parsi y cristiana y que provengan de Afganistán, Bangladesh y Pakistán (Government of India 2019). Notoriamente, no incluye a extranjeros pertenecientes a la comunidad musulmana, lo cual introduce un principio de discriminación religiosa que atenta contra los principios de la Constitución india.

El Registro Nacional de Ciudadanos (NRC, por sus siglas en inglés) fue creado en 1951 con base en los datos del censo de ese mismo año y como respuesta a la migración en el estado de Assam. El propósito era contar con un registro fidedigno de los ciudadanos en ese estado y así poder deportar a los extranjeros. Sin embargo, el registro no fue actualizado sino a partir de 2013, cuando la Suprema Corte comenzó a dirigir el proceso, y sus resultados fueron publicados en agosto de 2019. El problema es que el registro contenía los nombres de 31 de los 33 millones de habitantes de Assam, dejando fuera de la nación, literalmente, a 1.9 millones de personas.

Este texto está dividido en cinco secciones. En la primera se reflexiona brevemente sobre la seguridad humana. En la segunda se hace una introducción a la historia del

\footnotetext{
${ }^{2}$ Un ejemplo ofrece una idea de los cambios profundos emprendidos por el gobierno indio, que contribuyen a una frágil situación en términos de seguridad humana. El 5 de agosto de 2019, el gobierno indio anunció la decisión de eliminar el artículo 370 de la Constitución, que daba un estatus especial a Jammu y Kashmir (BJP, 2019). Asimismo, en octubre de ese año llevó a cabo una reorganización territorial por la cual se crearon dos territorios de la Unión: Jammu y Kashmir y Ladakh. En conjunto, esto alteró la autonomía de la cual gozaba ese estado y que fue uno de los elementos clave para su integración a India en 1947. Previo al anuncio de la eliminación del artículo 370 se enviaron tropas, se ordenó a los turistas que abandonaran el estado, se cortaron los medios de comunicación y se encarceló a líderes políticos. La población sufrió las consecuencias económicas y sociales de estas restricciones al movimiento y a la comunicación, a las que sumaron las que ocasionó la contingencia por la pandemia en 2020.
} 
movimiento nacionalista hindú, en particular del partido político BJP, en el poder de 2014 a 2019 y desde 2019 hasta ahora. En la tercera sección se introducen de manera sucinta algunas nociones sobre la Partición, en especial sobre ciudadanía y la migración que acarreó, así como sobre su impacto en Assam. En la cuarta y quinta secciones se exploran a profundidad los dos instrumentos antes mencionados. Finalmente, se abordan las implicaciones que para la seguridad humana presentan.

Para este texto se ha consultado la plataforma electoral del BJP en 2014 y 2019, pues allí hay elementos clave para analizar este proceso. Las leyes y sus enmiendas son parte importante y nos hablan de las especificaciones, objetivos y formas de comprensión de la nación india. Asimismo, se ha analizado documentación elaborada por el Estado: se ha echado mano del material, impreso y en línea, producido para los funcionarios a cargo, pues son quienes realizan el "proceso de traducción" y aplicación de las disposiciones para la población en general. Por ejemplo, el material para entrenar a los funcionarios locales que organizan reuniones comunitarias. Además se ha dado seguimiento a la prensa nacional e internacional, indispensable para entender la recepción de estos cambios.

\section{SEGURIDAD HUMANA}

En 1994 la Organización de las Naciones Unidas introdujo el concepto "seguridad humana", con el propósito de que la seguridad dejara de verse (sólo) como un tema propio de las relaciones entre Estados-nación y con énfasis en el ámbito militar. Se empezó a configurar una definición que comprendiera la dimensión humana de la seguridad: allí entraron la relevancia de la alimentación, la salud, el ingreso económico, el cuidado del ambiente para llevar una vida digna (Commission on Human Security 2003, 2-3). Durante la Reunión del Milenio de la ONU en 2000 se definieron los objetivos para el nuevo milenio, como la posibilidad de vivir sin miedo ni carencia. Estas ideas se han vuelto la expresión condensada de lo que engloba la seguridad humana (United Nations Fund Trust for Human Security).

Con el paso del tiempo, el término ha adquirido mayor peso y se han realizado estudios al respecto. Así, por ejemplo, se han estudiado las amenazas a la seguridad humana derivadas del deterioro ambiental. El cambio climático puede alterar profundamente las 
actividades económicas de las que dependen las poblaciones, provocando o profundizando la pobreza. Además, para los Estados resulta más difícil mantener la infraestructura pública (agua potable, educación o salud) debido a desastres naturales, pero también a las dificultades para recaudar impuestos (Barnett y Adger 2007). Como puede observarse, la investigación y la reflexión de este tipo no desplazan, más bien complementan la visión de la seguridad centrada en el Estado (Commission on Human Security 2003, 4).

Para el caso del Sur de Asia, y de India en particular, este cambio ha sido notable. En el caso de India, la definición inicial de seguridad partía de las amenazas al Estado indio: la integridad territorial, la autonomía en política exterior, el constituirse como una potencia regional y la estabilidad interna, como ilustra el libro de Muthiah Alagappa (1998). Allí figuraban de forma prominente la difícil relación con Pakistán y China y los movimientos de autonomía y secesión (Alagappa 1998).

Bajo la influencia de la definición dada por la Organización de las Naciones Unidas, un elemento común a este nuevo enfoque ha sido señalar la responsabilidad del Estado de garantizar la seguridad humana (Chatterjee 2014). Si observamos la región del sur de Asia en su conjunto, salta a la vista el enorme gasto militar realizado con el afán de obtener mayor seguridad, lo cual contrasta, irónicamente, con la permanente sensación de inseguridad de los gobiernos (Yousaf 2017, 486). Frente a esto, afirma Yousaf, es importante indagar en torno a problemas como la inseguridad económica, alimentaria y de salud, pues, aunque la región ha experimentado crecimiento económico, la pobreza y la incertidumbre siguen presentes. Un ejemplo concreto proviene del sector agrícola: cerca de $82 \%$ del campesinado es informal, el porcentaje más alto a nivel mundial. Debido a su condición, estos trabajadores son vulnerables y prácticamente incapaces de cubrir sus necesidades básicas ante la pérdida de empleo (Yousaf 2017, 488).

Adoptar la perspectiva de la seguridad humana permite tener una comprensión mayor de problemas sociales. Bashir Udin presenta un caso interesante: el del tráfico humano en la región, que ha sido estudiado en relación con el trabajo sexual y la migración: bajo el primer ángulo se exploran las condiciones de dicha actividad, y bajo el segundo la demanda y el flujo de personas que lo abastecen. Sin embargo, desde el punto de vista de la seguridad 
humana es posible ir más allá para entender que la pobreza es clave para el fenómeno en la región (Bashir Udin 2014).

El presente texto busca analizar la actualización del NRC y la Enmienda a la Ley de Ciudadanía a la luz de estas preocupaciones y amenazas a la seguridad humana. Para ello es importante entender la visión que alimenta ambos proyectos: el nacionalismo hindú, el cual concibe a India como un país puramente hindú, eliminando la diversidad que la caracteriza.

\section{EL NACIONALISMO HINDÚ EN EL PODER}

En el sur de Asia ha habido históricamente una serie de conflictos entre comunidades étnicoreligiosas. Estas disputas han estado asociadas a procesos diversos, como políticas coloniales, cambios en la posición social de una comunidad, nociones reales e imaginadas asociadas a mayorías y minorías, movimientos nacionalistas, construcción del Estado-nación y los esfuerzos de varios gobiernos por afianzar su posición en el poder. Los enfrentamientos durante la Partición, las confrontaciones entre musulmanes sunitas y chiítas en Pakistán, así como la persecución a los hindúes o budistas en Bangladesh son algunos ejemplos. ${ }^{3}$

Cabe destacar un rasgo de los gobiernos desde el periodo colonial hasta el poscolonial: la incapacidad para reaccionar y controlar la violencia contra las minorías religiosas. La inacción, la indiferencia y, a veces, incluso la participación de las fuerzas del orden constan en el pogromo de los sikhs en Delhi (India) en 1984, en los ataques en contra de musulmanes en Kalutara (Sri Lanka), así como contra los musulmanes en Delhi (India) en 2020.

El nacionalismo hindú en India tiene sus orígenes en el periodo colonial, como una más de las varias corrientes que participaron en el movimiento nacionalista (Hansen 1999). El nacionalismo hindú adoptó nociones de espiritualidad, misticismo y fascinación presentes en la imagen de India construida por el orientalismo del siglo XIX (Hansen 1999).

Ese nacionalismo construyó una visión propia del hinduismo, en la que ciertos elementos fueron privilegiados sobre otros, como la figura de Ram y la relevancia de los

\footnotetext{
${ }^{3}$ Estos conflictos étnico-religiosos no son exclusivos del sur de Asia, sino que están presentes también en otras zonas del continente. Desde los disturbios entre malayos y chinos en Malasia hasta la persecución de rohingyas en Birmania.
} 
lugares de peregrinación (dando lugar a una geografía sagrada). ${ }^{4}$ El movimiento fue desarrollando una visión particular de la historia y la cultura de India, en la que la centralidad de lo hindú se fue afianzando (Jaffrelot 1996; Van der Veer 1994).

Dos ideólogos importantes de este movimiento durante el periodo colonial fueron Vinayak Damodar Savarkar y M. S. Golwalkar. En su libro publicado en 1923, Savarkar introdujo un nuevo término: la Hindutva o hinduidad, que comprende lo hindú, pero más allá de la historia espiritual o religiosa, para abarcar la historia en su totalidad ([1923] 1969, 34). Savarkar delineó la historia de India identificando claramente los momentos de apogeo: la fundación de reinos por parte de los arios y la unificación realizada por Rama, así como también destacó la debilidad que acarreó el budismo y el declive a partir de la invasión musulmana ([1923] 1969).

A decir de Hansen, Savarkar desempeñará un papel relevante, pues sentará las bases para la construcción de un nacionalismo cultural que se distingue hasta el día de hoy por ser comunalista, masculino y antimusulmán. Sin embargo, también racionalista, pues, como nos recuerda Hansen, Savarkar admiraba el poder de los británicos $(1999,79)$.

Por su parte, durante la década de 1940, M. S. Golwalkar se encarga de desarrollar esta corriente. En We, Our Nationhood Defined (1939), establece que los hindúes son el elemento constitutivo de India. Para Golwalkar, aquellos que no se adaptan a los estándares de la nación hindú quedan fuera de ella, no merecen privilegios ni tratamiento especial, ni siquiera derechos ciudadanos (Hansen 1999, 80-84).

Durante el periodo colonial, la Hindu Mahasabha fue el partido político que asumió la defensa de la comunidad hindú, pero será hasta el periodo independiente cuando sobresalgan y se afiancen las estrategias organizativas del nacionalismo hindú en los ámbitos político y social. En la década de 1950, el partido Jana Sangh tuvo principios modestos en la vida electoral de India; si bien sus resultados no fueron impresionantes, destacó la movilización de integrantes de la Rashtriya Swayamsevak Sangh (RSS) o Asociación

\footnotetext{
${ }^{4}$ El dios Ram y la construcción de un templo dedicado a él han sido objetos de movilización política para el grupo de organizaciones políticas, sociales y culturales del nacionalismo hindú. El templo se construirá sobre el terreno que alguna vez ocupó la Mezquita de Babar, que fue arrasada en 1992 por estos grupos y sus simpatizantes.
} 
Nacional de Voluntarios, que se caracterizó por el llamado a fortalecer la comunidad hindú (Jaffrelot 1996; Hansen 1999).

Es hasta la década de 1980 cuando integrantes del Jana Sangh fundan un nuevo partido, el BJP, que se asume como sucesor del anterior. Al cabo de esa década y durante la de 1990, el BJP cobrará mayor relevancia política a nivel nacional, tratando además de enlazarse con preocupaciones regionales y locales específicas.

A finales de la década de 1990, el BJP adquirió una fortaleza mayor aún. Durante esa década, varios gobiernos se formaron con base en frágiles alianzas. El propio BJP formó un gobierno de corta duración en 1996, pero en el periodo de 1998 a 2004 logró permanecer en el poder durante cinco años, con Atal Bihari Vajpayee como primer ministro.

Si bien el Partido del Congreso retomó el poder en 2004 y 2009, en 2014 el BJP regresó. Para entender esta victoria política, hay varios elementos a considerar, como la redefinición de la figura pública de Narendra Modi, después de su pobre desempeño durante la violencia en Gujarat en 2002; la presencia de importantes líderes políticos del BJP en varios estados, los problemas económicos del país y las acusaciones de corrupción dirigidas al Partido Nacional del Congreso, entre otros más.

Las elecciones de 2014 son notables por varias razones. Desde 1984 un partido político no había logrado ganar los votos suficientes por sí mismo. El BJP encabezó una alianza denominada Alianza Democrática Nacional (NDA, por sus siglas en inglés), pero por sí solo obtuvo suficientes escaños en el Parlamento. Narendra Modi asumió el cargo de primer ministro, lo cual supuso una transformación de su figura política después de ser jefe de gobierno del estado de Gujarat.

Durante su largo paso al frente de Gujarat (desde 2001 hasta 2014), Modi demostró su incapacidad para lidiar con la violencia, pero también su falta de sensibilidad hacia la comunidad musulmana. En febrero de 2002, un carro del tren donde viajaban peregrinos hindúes que regresaban de Ayodhya se incendió. El tren se había detenido en la estación de Godhra, Gujarat, cuando comenzó el incendio. Al día de hoy, las causas no han sido aclaradas, pero los peregrinos dieron una versión según la cual habría ocurrido una pelea con musulmanes que estaban en la estación, quienes habrían prendido fuego al vagón a manera 
de venganza (Martínez Saavedra 2016). Días después se organizó una persecución de musulmanes, en la que participaron, entre otros, organizaciones nacionalistas hindúes, como la RSS. Se calcula que 1000 personas murieron, aunque hay algunos que mencionan la cifra de 2000 muertos (Martínez Saavedra 2016).

La actuación de Modi fue deplorable, pues en vez de asegurarse de que las fuerzas del orden intervinieran, hubo negligencia e incluso acusaciones de que miembros de la policía habrían participado en los ataques. Adicionalmente, Modi se expresó de forma irresponsable al declarar que a toda acción corresponde una reacción, es decir, que la persecución de los musulmanes era resultado del incendio del tren (“'Newton’ Modi has a lot to answer” 2002). El propio Atal Bijari Vajpayee, primer ministro, tardó en pronunciarse y lamentar lo ocurrido, lo que fue objeto de muchas críticas.

Si los señalamientos ante lo ocurrido fueron numerosos, vienen a la mente dos preguntas: ¿cómo es que Modi dejó atrás esta imagen para asumir el cargo de primer ministro en 2014 y nuevamente en 2019? Y ¿cómo es que el BJP volvió al poder, después de perder ante el Partido del Congreso en 2004?

Para responder a estas interrogantes, en primer lugar, es preciso analizar la transformación de Modi. Su imagen pública se enfocó en otros elementos durante su paso por la gubernatura. Se destacó su impulso a los empresarios, quienes se vieron favorecidos y alabados, lo cual contrasta con su falta de interés en sectores pobres. Se habló del crecimiento económico logrado, que, sin embargo, venía de tiempo atrás y no se debía exclusivamente a él. Se enfatizaron sus promesas de una India pujante, con avances tecnológicos (Jaffrelot 2015; Martínez Saavedra 2016).

Esto estuvo acompañado de la exposición constante de su imagen. Para empezar, Modi contrató a una compañía estadunidense de relaciones públicas, además de explorar otras tecnologías, como el correo electrónico (respondía personalmente parte de sus correos) y los mensajes a celular (Jaffrelot 2015,3). Asimismo, fue sumamente cuidadoso al elegir la ropa, los colores y la postura que asumía en cada fotografía, lo mismo que el tono de voz y el lenguaje corporal en sus apariciones públicas (Jaffrelot 2015, 4-5). Estos elementos fueron recuperados y profundizados a nivel nacional durante las campañas políticas de 2014 y 2019. 
En segundo lugar, la lectura detenida de la plataforma electoral del BJP para 2014 y su contraste con la plataforma de 2019 es sumamente útil para comprender los elementos clave en cada coyuntura electoral, en específico, la centralidad que fue adquiriendo la definición de India como hindú, a partir de proyectos concretos. Al comparar ambos documentos se notan diferencias importantes. Hay que tomar en cuenta el contexto. En 2014, el BJP estaba tratando de regresar al poder, por lo que tomó como punto de partida la crítica al gobierno del Partido del Congreso.

El texto consta de 42 páginas. Sus subtítulos muestran un plan de trabajo claro, que convoca a construir una India fuerte. Así nos encontramos con el llamado a "atender lo inminente", es decir, todos los problemas heredados por el Partido del Congreso, entre ellos, la corrupción, el alza de los precios y la crisis de credibilidad. Posteriormente se invita al electorado a participar en "fortalecer la estructura", "reformar el sistema" y "ampliar la plataforma". La agenda concluye con el compromiso de erigir una India vibrante, con una sociedad y economía del conocimiento (BJP 2014, 42.)

Sin embargo, temas que se volverán centrales hacia 2019 y que revisten relevancia para nuestro análisis, reciben breves menciones. El documento establece que es importante garantizar el desarrollo regional y que en el noreste indio hay varios temas pendientes. Entre ellos está el de atender el problema de los infiltrados en el noreste y ponerles un alto, además de establecer lineamientos claros (BJP 2014, 8).

En cuanto a Jammu y Kashmir, el BJP se comprometía a mantener la unidad territorial india y a derogar el artículo 370, entre otras cosas (BJP 2014, 8).

Hacia el final, el documento reflexiona sobre la presencia de graves problemas en términos de seguridad nacional, ampliamente definida, que incluyen lo mismo capacidad militar que seguridad económica y acceso al agua (BJP 2014, 37). Debido a errores de administraciones pasadas, aduce el BJP, problemas importantes no han sido atendidos. La región es sensible y hay problemáticas internas, como asuntos en la Marina, disturbios comunistas, ataques maoístas y la presencia de inmigrantes ilegales en el noreste (BJP 2014, 38). 
El propio BJP resume en un par de listas ideas clave para su posible administración. La primera lista lleva como título "Nuestro compromiso", con énfasis en el proyecto para el electorado: crecimiento económico, combate a la corrupción, competitividad en el plano internacional y sistemas de gobernanza transparentes, en los que haya realmente rendición de cuentas. Al final del manifiesto, la segunda lista tiene un título engañoso: "Legado cultural", pues el lector anticipa preocupaciones como asegurar fondos para el Archeological Survey of India (para sus museos, biblioteca y publicaciones), pero en realidad se encuentra con una lista de compromisos para preservar las lenguas indias y los sitios históricos, así como la protección de la vaca y su progenie, la construcción del Templo de Ram y la limpieza del río Ganges, pues es un símbolo de fe. Inmediatamente abajo, sin relación directa con estos temas, se enuncia un compromiso adicional: el establecimiento de un Código Civil Uniforme, para lograr la equidad de género (BJP 2014, 41). No hay elemento de sorpresa en los temas listados: todos y cada uno responden a la idea de lo hindú como lo único valioso y digno de cuidado para la nación. La única sorpresa, quizá, resida en que esta lista no aparezca como introducción, sino al final, para suavizar el tono y disminuir la percepción entre el electorado de que el BJP alienta las divisiones al interior de la sociedad india.

Sin embargo, para 2019 y después de un periodo en el poder, la agenda electoral reflejaba un tono de mayor confianza. Esto era evidente tanto en la organización del documento, como en la centralidad de algunos temas. Esta vez el documento comenzó con algunos mensajes breves de Modi, primer ministro; Amit Shah, presidente del BJP, y Rajnath Singh, quien encabezó el comité que elaboró este manifiesto, más un apartado llamado "Hacia una nueva India” (BJP 2019, 2-10).

En esta ocasión prescindieron de una introducción como tal y presentaron un primer apartado titulado "La nación primero", que en 14 puntos delineó su visión de India. Figuran allí dos grandes grupos de ideas, uno vinculado con la seguridad nacional entendida como un tema de defensa, de allí que se hable de combatir el terrorismo, asegurar las fronteras y garantizar el bienestar de los soldados. Otro en que se delinea la idea que hay de la nación y que contiene dos elementos relevantes para nuestro tema: se combatirá la infiltración que atenta contra la identidad cultural y lingüística en ciertas zonas. Para ello se llevará a cabo la actualización del Registro Nacional de Ciudadanos, además de reforzar la vigilancia en la 
frontera. Asimismo, se implementará la enmienda a la Ley de Ciudadanía, para recibir refugiados de minorías comunitarias de países vecinos. Pero, aclaran rápidamente, sin menoscabo de la identidad cultural y lingüística del noreste indio. La promesa de cambiar el estatus de Jammu y Kashmir también figuró, pero como una forma de asegurar el desarrollo y de proteger a ciertos sectores (BJP 2019, 11-12).

Las elecciones se celebraron entre abril y mayo de 2019. De allí salieron victoriosos el BJP y su alianza, la Alianza Democrática Nacional (NDA) (Mehta, 2019b). El análisis de las primeras acciones y comunicaciones de un gobierno electo arroja generalmente ideas sobre lo que viene, permite anticipar, si se quiere, el tono de la administración. El BJP asumió el poder en agosto y pocos días después derogó el artículo 370 relacionado con Jammu y Kashmir (Mehta 2019a). El mensaje inequívoco era que, efectivamente, la nación (como el BJP la concibe) venía primero y que cumplirían las promesas hechas.

\section{CIUDADANÍA, ETNICIDAD, LENGUA Y FRONTERA}

Para entender el origen tanto del Registro Nacional como de la enmienda a la Ley de Ciudadanía es preciso remontarse al periodo colonial y examinar, por un lado, lo ocurrido en Assam, provincia británica ubicada en el noreste. Y, por otro, hacer algunas observaciones sobre la construcción de las fronteras al momento de la independencia, así como a algunos arreglos estatales de Bangladesh, India y Pakistán. Es en torno a estos procesos y lugares como se fueron construyendo nociones de ciudadanía y pertenencia, de responsabilidad estatal y de los que surgieron temores ante la presencia de gente foránea (los "extranjeros ilegales" del periodo poscolonial). A continuación, iré entrelazando algunas reflexiones sobre la situación de Assam, con el proceso más amplio de Independencia y Partición. No se trata de una explicación cronológica en sentido estricto, sino de una exposición que busca problematizar estos procesos.

Baruah ha dicho que en el tratamiento dado a Assam durante el periodo colonial no hubo consideraciones de carácter cultural o histórico, sino económicas. Los británicos se apropiaron de Assam en la primera mitad del siglo XIX y la administraron como una extensión 
de la provincia de Bengala, hasta que finalmente en 1874 adquirió un estatus separado (chief commissionership) (Baruah 2001, 24-25).

Al interior de Assam, la población era diversa e incluía lo mismo a hablantes de asamés que a hablantes de otras lenguas, pertenecientes a tribus. Además de ello, los hablantes de bengalí y de lenguas adicionales crecieron durante el siglo XIX y el XX. Por un lado, Sylhet, un distrito con mayoría bengalí era parte de Assam, lo que alteró la composición demográfica a favor de la población musulmana. Por otro, diversas actividades económicas impulsaron la llegada de migrantes de diversos orígenes, entre ellos bengalíes, tanto hindúes como musulmanes. Los británicos alentaron la importación de mano de obra de Mymensingh, Rangpur y otros lugares, para que trabajara en el tendido de vías férreas. Posteriormente, con el establecimiento de la industria del té, llegaron miembros de las tribus santhal, oraon, munda. Otro grupo importante fue el de los nepalíes: muchos de ellos se emplearon en el sector de servicios y fueron trabajadores en la construcción, el transporte y la agricultura (Baruah 2001, 44-68; Hazarika 2000, 71-74).

La percepción entre los británicos de que el asamés era un derivado del bengalí, aunada a la presencia de bengalíes, hicieron que el idioma bengalí se convirtiera en el medio de comunicación de las cortes y en el idioma oficial desde 1837 hasta 1873 . Esto generó un movimiento entre los intelectuales, quienes defendieron su lengua y, con el apoyo de misioneros cristianos, quienes estaban elaborando materiales que ayudaran en su labor de conversión, lograron que el asamés se convirtiera en la lengua de la provincia (Baruah 2001, 38-39). Estos elementos explican el origen del temor de la población asamesa a volverse minoría frente a la población hablante de bengalí.

Los miedos se incrementaron en la segunda mitad del siglo XX, a consecuencia de una mayor migración. Es importante recordar que, en los últimos años del periodo colonial, la confrontación entre fuerzas políticas (el Partido Nacional del Congreso y la Liga Musulmana) y ciertos elementos de la política colonial condujeron a la independencia de la colonia, pero con una división territorial (Carballido Coria 2011). A grandes rasgos, ambos partidos políticos tenían visiones encontradas: mientras que el Partido del Congreso insistía en asumirse como el representante de todos los indios y en vislumbrar una India con un gobierno centralizado, la Liga Musulmana mostraba preocupación por la posición de los musulmanes 
en una India independiente y aspiraba a obtener mecanismos que garantizaran su seguridad, para lo cual un sistema federal era lo mejor. No obstante, estos elementos se complejizaban a nivel regional y local con elementos de orden económico, social y político (Carballido Coria 2011).

Finalmente, India se dividió en dos: India, como la conocemos ahora, y Pakistán, formado a partir de las zonas al oeste y este que tuvieran mayoría de población musulmana y continuidad geográfica. En 1947 Pakistán tenía una peculiaridad: era un país compuesto por dos grandes territorios carentes de continuidad geográfica y con otro país en medio de ellos. No obstante, esta situación no sería permanente, pues en 1971 la parte este se transformaría en Bangladesh.

Después de la Independencia y Partición de India en dos Estados, India y Pakistán, continuó la migración. Tras dividirse, se registró un intercambio poblacional particularmente agudo (pero no restringido, como veremos) en los pueblos cercanos a las nuevas fronteras entre los dos países. Así, personas no musulmanas (hindúes y sikhs) decidieron emigrar a India al encontrarse dentro de Pakistán y personas musulmanas decidieron emigrar a Pakistán (Butalia 1998).

De la parte este del recién creado Pakistán llegó a Assam población hindú que huía de la violencia comunalista. A la vez, personas de otros estados de la República india, pero particularmente de Bengala en India, continuaron llegando a Assam en busca de mejores condiciones de vida. A estos flujos se añadió el de 1971, cuando Pakistán del este se independizó de Pakistán del oeste y se convirtió en Bangladesh: una cantidad importante de refugiados hindúes cruzó la frontera hacia India debido a la violencia y muchos de ellos terminaron en Assam.

De acuerdo con Baruah, las movilizaciones sociales se organizan en torno a símbolos culturales, y en el caso de la población originaria de Assam su movimiento ha extraído dichos símbolos de leyes y de la Constitución $(2001,118)$. Las protestas en contra de la presencia de "extranjeros ilegales" se han organizado en buena medida en torno a las fechas a partir de las cuales se cierra la nación y ya no es posible aceptar más migrantes. 
Pero la respuesta a la interrogante de quién está o no dentro de la nación no ha sido sencilla ni en el sur de Asia ni en el resto del continente. Al independizarse, ni India ni Pakistán eran (y siguen sin ser) países homogéneos en términos religiosos, lingüísticos o étnicos. La conciencia de esto es palpable en los escritos previos a la Partición, cuando intelectuales y líderes se plantearon el problema: ¿sería pertinente realizar un intercambio poblacional entre los dos países?, ¿las comunidades minoritarias en Pakistán funcionarían como "rehenes" que garantizarían el bienestar de las comunidades minoritarias en India y viceversa? (Carballido Coria 2011; Chatterji 1996; Talbot 1998). Estas preguntas estaban contenidas en textos que circularon en favor y en contra de la creación de Pakistán (Ahmad 1947; Chatterjee 1947).

Las personas huyeron de la violencia y la incertidumbre. La violencia comunalista empezó en Bengala en agosto de 1946, a raíz del Día de Acción Directa, y se extendió a otras zonas de la colonia. Asesinatos, violaciones, conversiones forzadas, saqueos, desacralización de espacios se sucedieron a partir de ese momento y hasta fines de 1948 (Butalia 1998; Menon y Bhasin 2000). En particular, una vez que se anunció la frontera final entre los dos países, los movimientos de población se aceleraron, pero con el tiempo adquirieron patrones diferentes. En este aspecto, especialistas de la Partición han señalado que mientras una gran cantidad de personas se trasladó hacia el lado oeste en un periodo más corto, el movimiento poblacional fue menos numeroso hacia el este, con picos en momentos específicos, pero continúa prácticamente hasta el día de hoy (Bagchi y Dasgupta 2006; Butalia 1998; Menon y Bhasin 2000; Talbot 1998).

Lo cierto es que ninguno de los dos países estaba preparado para la cantidad de inmigrantes que recibió ni para los arreglos necesarios que esto conllevó: desde la organización de campamentos y la recuperación de personas que habían sido robadas (mujeres y niños), pasando por cuestiones de propiedad, hasta la decisión de la fecha final para este flujo migratorio (Butalia1998; Menon y Bhasin 2000; Talbot 1998). Pakistán, por ejemplo, recibió 95000 refugiados tan sólo de Hyderabad, como consecuencia de la "acción policial" de 1948 del gobierno indio (Talbot 1998, 105). Por su parte, el gobierno indio anunció que el 19 de julio de 1948 sería el último día para que los pobladores provenientes de Pakistán reclamaran la ciudadanía india. Pero, posteriormente, como resultado del Pacto 
Nehru-Liaquat, concerniente a la protección de minorías en ambos países, la fecha se recorrió al 31 de diciembre de 1950 (Chandra et al. 2002, 81). Sin embargo, en la práctica, los cruces continuaron, pues el sistema de pasaportes se introdujo hasta 1952, y, como hemos visto, continúan hasta hoy.

En Assam, las protestas en contra de la presencia de "extranjeros ilegales" se volvieron cada vez más prominentes desde el inicio de la independencia. No obstante, la identificación de esos extranjeros no era fácil: la creación de India y Pakistán dividió comunidades que hasta hacía no mucho tiempo tenían lazos a ambos lados de la nueva frontera: bengalíes que habitaban en Calcuta (ahora Kolkata), cuyo hogar ancestral se había quedado en Pakistán del este. O trabajadores agrícolas que iban y venían entre Bengala y Assam. Para determinar quién era extranjero y quién no, se hizo el primer Registro Nacional de Ciudadanos: en éste se pusieron los datos particulares de las personas incluidas en el primer censo, realizado en 1951. Sin embargo, el Registro Nacional no volvió a actualizarse, sino hasta años recientes.

Sanjoy Hazarika inicia un par de sus libros sobre el noreste indio con historias que ilustran parte de estos movimientos migratorios hacia India (1995, 2000). Sheik Barah, un sastre, vino de Pakistán del este (Bangladesh) con su esposa en 1971, antes de que el ejército indio entrara a liberar el país. En 1984, a los 45 años, su nombre fue registrado como un elector del basti, legalizando su presencia (Hazarika 1995, 4-9). Mahesh Chandra Das, un bengalí hindú originario de Sylhet, era el cocinero de la familia del autor. Mahesh tenía algunas peculiaridades, entre las que figuraban el preparar un delicioso ilish (hilsa curry o curry de pescado), fumar biris malolientes y cruzar cada año la frontera hacia Pakistán del este, que eventualmente se convertiría en Bangladesh. Lo notable es que Mahesh emprendía el cruce sin documentos: al irse, entregaba un soborno al guardia de Pakistán del este, y al regresar, uno al guardia de India (Hazarika 2000, 9-15). Mahesh lograba así un equilibrio y mantenía la paridad, parafraseando a Hazarika $(2000,12)$.

Hay especulaciones y debates en torno a la cifra de migrantes, ejemplificados en estas dos historias. En Assam se hablaba de que los extranjeros constituían 31\% a 34\% de la población para 1971 (Baruah 2001, 118). En la década de 1990 se hablaba de tres millones de bengalíes en Delhi (Hazarika 1995, 4-9). En 2004, el ministro de Estado para Asuntos del 
Interior, Sriprakash Jaiswal, dijo en el Parlamento que había 12 millones de bangladeshíes ilegales en India (India and Bangladesh. Migration Claims Fact-Checked 2020). Sin embargo, en un texto de 2012 sobre la migración sur-sur, las Naciones Unidas señalaron que el número de migrantes internacionales de Bangladesh que reside en India era de 3200000 (Organización de las Naciones Unidas 2012, 3).

Las condiciones de vida en Bangladesh, aunadas al deterioro ambiental están detrás de esto (Hazarika 1995, 4-9). Sin embargo, también hay otros factores que lo explican, como la cercanía geográfica, la cultura y el pasado compartidos.

Hacia el final de la década de 1970, en Assam se registró una amplia movilización en contra de los inmigrantes: el incremento de hablantes de lengua bengalí (que traía a la memoria eventos del siglo XIX), así como la presencia de "extranjeros" en las listas de votantes fueron elementos prominentes. La movilización se prolongó hasta 1985 e incluyó actos de violencia, de los cuales el más notable fue la masacre de Nellie, en la que poco más de 2000 personas fueron asesinadas (Baruah 2001, 132-135; Hazarika 2000, 49-53).

Esta agitación concluyó con la firma de un acuerdo entre el movimiento y el gobierno indio, el cual implicaba la organización de los inmigrantes en tres categorías. Quienes hubieran ingresado antes de enero de 1966 serían considerados ciudadanos, pero quienes hubieran entrado entre enero de 1966 y marzo de 1971, pese a poder ser considerados ciudadanos, serían eliminados de las listas electorales e inhabilitados para votar durante 10 años. Quienes hubieran llegado después de 1971 serían considerados extranjeros y serían deportados (Baruah 2001, 138-139). ${ }^{5}$ La elección de la fecha de marzo de 1971 no es casual: ese mes había surgido una nueva nación, Bangladesh.

No obstante, las protestas y acciones en contra de los inmigrantes al interior de Assam no terminaron con el acuerdo. El flujo migratorio ha continuado, en buena medida, por razones económicas, aunque no únicamente. Por ello, en Assam se recibió con beneplácito la noticia de que la Suprema Corte vigilaría el proceso de actualización del registro en ese

\footnotetext{
${ }^{5}$ Esta disposición da pie a varias preguntas y señala contradicciones en la presencia de estos migrantes calificados como “extranjeros ilegales": ¿cómo es que llegaron a ser incluidos en las listas electorales?, ¿después de tanto tiempo habitando y trabajando en India no podrían ser considerados nacionales? Claramente, los Estados-nación alrededor del mundo establecen etapas para la obtención de la ciudadanía por naturalización, pero el derecho al voto suele ser un atributo junto con otros de la ciudadanía plena, mientras que el derecho al voto para los no-ciudadanos suele operar bajo condiciones extraordinarias.
} 
estado durante 2014 y 2015, con la esperanza de que ello concluyera con la deportación de los extranjeros. Como hemos mencionado, el gobierno indio tiene contemplado implementar el Registro Nacional de Ciudadanos para todo el país en 2021.

\section{Registro Nacional de CiUdAdanos: LA PUESTA EN MARCHA}

Como hemos mencionado, la Suprema Corte monitoreó el proceso de actualización del registro en el estado de Assam. Para llevarlo a cabo, el gobierno estatal diseñó una compleja serie de pasos (tabla 1). En primer lugar, publicó los datos patrimoniales (Legacy Data) en los centros de atención (National Registration Seva Kendra) y oficinas electorales, además de hacerlo en su página web: aquéllos consistían en el Registro Nacional de 1951 y las listas electorales hasta 1971. Todos los habitantes de Assam debían buscar ahí su nombre o el de su padre, madre o ancestro. Posteriormente anotarían varios datos, que proporcionarían al funcionario del centro de atención. Con base en ellos, el funcionario proporcionaría a las personas un talón con un código único (Legacy Data Code) (Government of Assam 2014).

El ciudadano debía anotar el código en su formato de solicitud para ser incluido en el Registro Nacional de Ciudadanos. Los formatos a llenar estarían disponibles en inglés, asamés y bengalí. Si el ciudadano aparecía en alguna de las listas, debía probar su parentesco con los familiares a quienes deseaba incluir en el Registro Nacional. Si, por el contrario, quien aparecía en alguna lista era su ancestro, entonces debía establecer su parentesco y el de sus descendientes. Los documentos posibles a presentar constituían actas de nacimiento, certificados universitarios y registros de propiedad, entre otros más.

TABLA 1. Etapas de la actualización del Registro Nacional de Ciudadanos

\begin{tabular}{ll}
\hline \multicolumn{1}{c}{ Fase } & \multicolumn{1}{c}{ Periodo } \\
\hline $\begin{array}{l}\text { Publicación de datos patrimoniales } \\
\text { Fase de distribución y recepción de formatos de } \\
\text { solicitud }\end{array}$ & $\begin{array}{l}\text { 27 de febrero al 31 de agosto de 2015 } \\
\text { Recepción de solicitudes y documentos hasta el } 31 \\
\text { de agosto de } 2015\end{array}$ \\
$\begin{array}{l}\text { Fase de verificación } \\
\text { Publicación del borrador del NRC }\end{array}$ & $\begin{array}{l}\text { A partir del } 1^{\circ} \text { de septiembre de 2015 } \\
\text { Fase de publicación del borrador Completo del NRC diciembre de } 2017\end{array}$ \\
$\begin{array}{l}\text { y recepción de demandas y objeciones } \\
\text { Publicación final del NRC }\end{array}$ & 31 de agosto de 2019 \\
\hline
\end{tabular}

FUENTE: Elaborada con base en Government of Assam (2014c). Timelines Chart. 
Posteriormente habría un periodo de verificación. En las oficinas se revisarían las solicitudes y los documentos adjuntos. En los recorridos se haría una verificación cuasi judicial para identificar a las personas y establecer la veracidad de sus solicitudes de ser incluidas en el NRC (Government of Assam 2014b).

Ésta no era la primera vez que en el periodo independiente recaía en el ciudadano la responsabilidad de demostrar quién era y/o su pertenencia a ciertos grupos. Un ejemplo conocido es la protección de las Castas Registradas (intocables), principalmente en términos de representación y de reservación de cuotas de empleo, así como en el sistema educativo. En cuanto al primer punto, la Constitución introdujo la representación en las Asambleas Legislativas de la Unión y de los estados de acuerdo con su población. En cuanto al segundo, se reservaron empleos dentro del sector público y las escuelas, siempre y cuando el candidato estuviese calificado para ello. Es importante aclarar que las Tribus Registradas (grupos tribales) también se vieron beneficiadas con la reservación de cuotas.

En ambos casos de reservación de cuotas, el Estado indio ha elaborado listas con los nombres de castas y tribus. Así, para que una tribu sea reconocida debe demostrar que posee varios atributos, lo cual pasa por el ámbito de la escritura pues hay que enviar un grueso expediente, pero también por el corporal, pues hay que montar una exhibición convincente. Townsend Middleton ha hecho una detallada (y divertida) descripción de esto, cuando una tribu de Darjeeling decidió hacer una solicitud y recibió la visita del funcionario gubernamental. Ser parte de una tribu implica demostrar pureza cultural y en esta instancia significaba demostrar que eran budistas sin “influencia hindú” alguna (Middleton 2015).

Sin embargo, el Registro Nacional de Ciudadanos introdujo una nota distinta, cargada de gran incertidumbre. Esta vez los ciudadanos debían demostrar que sí lo eran, es decir, el punto de partida consistía en la sospecha de que se era un invasor.

A lo largo del proceso de solicitud las dificultades fueron enormes. En primer lugar, estaba la barrera de la literacidad. India posee una tasa de alfabetización que, si bien se ha elevado, todavía presenta deficiencias. Si tomamos la población joven (15 a 24 años), 81\% puede leer y escribir, pero si dividimos la población en hombres y mujeres el porcentaje es de $88 \%$ y $74 \%$ respectivamente. Sin embargo, si abarcamos la población adulta en su totalidad (15 años en adelante), entonces el porcentaje es inferior: el total es de 63\%, mientras 
que para hombres es de 75\%, y 51\% para las mujeres. Estas cifras corresponden a 2005-2010 (Unesco 2013, 48).

El llenado de los formatos y las detalladas instrucciones para ello podían consultarse impresos o en línea. Además se organizaron reuniones con funcionarios locales, quienes se encargaron de "entrenar" a la población y de "sensibilizarla" en el laberinto de las solicitudes para el registro (Government of Assam 2014d).

En segundo lugar, un país donde es frecuente la falta de documentos de identificación, la presentación de un acta de nacimiento o de matrimonio constituyó una tarea difícil.

En tercer lugar, seguir el largo proceso implicó tiempo y recursos. Si bien los ciudadanos podían acudir a los centros de atención y allí obtener los formatos de forma gratuita, tenían que presentar los documentos probatorios fotocopiados. En la etapa de verificación podían presentar los documentos originales o mostrarlos durante alguna de las visitas de campo. Finalmente, para averiguar los resultados era posible acudir a los centros de atención o buscarlos en la página web, pero la brecha digital en India no lo hacía posible.

Por si esta descripción del intricado proceso no fuera suficientemente desalentadora, la desigualdad de género se tradujo en problemas adicionales. Como hemos señalado, la tasa de alfabetización es más baja entre las mujeres, además de que la dependencia económica y social, reflejada en una participación menor en la población económicamente activa, hicieron que el proceso para las mujeres fuera más penoso (Unesco 2013). Es importante agregar que las mujeres debían probar su ciudadanía ubicándose ellas mismas o sus ancestros en la documentación de los datos del legado, no a través del esposo. Si bien esto puede parecer aceptable, para muchas mujeres habría sido más fácil presentar un acta de matrimonio que un acta de nacimiento con los nombres de su padre y madre.

Finalmente, el 31 de agosto de 2019 se publicó la lista final de ciudadanos: 31.1 millones de pobladores de Assam fueron incluidos como ciudadanos, mientras que 1.9 millones quedaron fuera. Inicialmente se habían revisado alrededor de 33 millones de solicitudes y se habían dejado fuera cuatro millones, de los cuales se hizo una revisión y se publicó la lista final. 
Historias angustiosas $-\mathrm{y}$ absurdas - abundan al revisar los medios noticiosos nacionales e internacionales. Hubo familias cuyos integrantes estuvieron en la lista "a medias", es decir, estuvieron uno de los progenitores y algunos hijos, pero no el otro progenitor y el resto de los hijos. Como resultado de lo que se ha explicado, para muchas mujeres eran previsibles situaciones particularmente difíciles (India excludes nearly 2 million from Assam citizen list 2019; How Fate of 19 lakh people hang hangs in balance in Assam after NRC's final list 2019).

Hubo un caso muy notorio de un veterano de guerra que no estuvo ni en la primera ni en la segunda versiones del registro y que permaneció 10 días en un centro de detención (Assam NRC. What Next for 1.9 million stateless Indians? 2019).

Otras tantas historias conciernen a diferencias ortográficas o confusiones en los nombres. Fueron numerosos los casos de personas cuyas solicitudes fueron rechazadas porque había una discrepancia en la escritura de su nombre en los documentos presentados. También hubo casos de personas cuyo nombre fue confundido con el de alguien más (India excludes nearly 2 million from Assam citizen list 2019).

Las personas que no estuvieron en la lista contaban con 120 días para apelar. Debían presentarse ante Tribunales de Extranjeros, organismos cuasi judiciales que, se esperaba, responderían en un plazo de seis meses. Pero la forma de operar de estos tribunales ha sido descrita como arbitraria: para muchas personas es difícil presentar evidencia que permita la identificación (no poseen documentos), además de que, como hemos señalado, diferencias en la forma de escribir un nombre o en la fecha de nacimiento pueden llevar a la conclusión de que es una persona inmigrante ilegal.

En la segunda mitad de 2019 hubo incertidumbre sobre la suerte que correrían aquellos que no estuvieron en la lista. Personas que no aparecieron en los borradores previos de la lista invirtieron tiempo y recursos para obtener ayuda legal y poder presentar nueva documentación. Acaso acudir a los Tribunales para Extranjeros, no implica admitir que no se es ciudadano. Asimismo, hay seis lugares de detención en Assam, en los que se encuentran personas sospechosas de ser extranjeras, pero su presencia allí no está relacionada directamente con el NRC. 
Un artículo del Hindustan Times resumió así lo ocurrido:

El Registro Nacional de Ciudadanos ha sido actualizado, excluyendo un poco más de 1.9 millones de personas (6\% de la población de Assam). El proceso del NRC tomó cuatro años, involucró un despliegue masivo de maquinaria burocrática, se volvió uno de los temas políticos más contenciosos en India contemporánea, profundizó las divisiones existentes en la sociedad de Assam y produjo un resultado con el que nadie está contento (NRC An Experiment Gone Awry 2019).

\section{ENMIENDA A LA LEY DE CiUdAdANÍA (2019)}

En 1946, durante las negociaciones por la independencia se formó la Comisión Constituyente, que continuó su trabajo al independizarse el país en 1947. Al término de sus labores se adoptó la Constitución, el 26 de noviembre de 1949, y entró en vigor el 26 de enero de 1950. En sus XXII partes la Constitución contenía elementos de avanzada para esa época, que siguen vigentes al día de hoy.

Durante el proceso de conformación del Estado indio se han detectado tanto continuidades como rupturas con el régimen colonial, así como esfuerzos importantes por sentar las bases de un sistema democrático. Paul Brass ha destacado entre las continuidades el modelo federal y la división de tareas legislativas en tres esferas: el Centro, las provincias y una en que participan ambos; así como la presencia de los funcionarios públicos de carrera que daban soporte a la administración colonial (Indian Civil Service, ICS) y continuaron dándolo ahora al Estado independiente (Indian Administration Service, IAS 2004, 2).

Entre las rupturas, Brass ubica la introducción de derechos fundamentales, el sufragio universal, programas permanentes de desarrollo económico y social, además de una serie de medidas para evitar que el Estado impacte negativamente sobre el ejercicio de los derechos de los ciudadanos (2004, 2-3).

Estas ideas son fruto de la contribución de un brillante grupo de políticos, entre los que encontramos a Gandhi, Nehru, Patel y Ambedkar. La visión de India que tenían ellos, nos dice Sunil Khilnani, era la de una India diversa y plural. Esto se tradujo en derechos, en una vida democrática no sin sus problemas y con espacio para el disenso (2004). No obstante, 
la visión que el BJP tiene de India pone en peligro esa construcción: empobrece la nación, ya que excluye todo aquello que no es hindú.

Cuando en 2016 el BJP presentó un proyecto de ley que concedía la ciudadanía india a hindúes, sikhs, jainas, budistas, parsis y cristianos provenientes de Bangladesh, Pakistán y Afganistán que hubieran entrado al país sin documentación, puso de manifiesto un esfuerzo para proteger a esas minorías, como un recurso humanitario para atender a refugiados (BJP 2019, 12).

La enmienda fue aprobada en diciembre de 2019 y generó innumerables críticas y preguntas. Su carácter excluyente es visible y no logra esconderse bajo el argumento de la protección a minorías perseguidas, pues un vistazo rápido a la región del sur de Asia y a regiones adyacentes obliga a preguntarse ¿por qué sólo esos países y esas comunidades son mencionadas?, ¿qué hay de la vulnerabilidad de los rohingyas en Birmania? Si Sri Lanka también tiene minorías oprimidas, ¿cómo es que no está incluida?, ¿acaso no hay comunidades musulmanas en Pakistán que también son reprimidas?

Asimismo, innumerables protestas (aunque no por las mismas razones) se efectuaron a lo largo y ancho del país. La mayoría de ellas se centró en el hecho de que la enmienda atenta contra la Constitución, pues introduce un elemento de discriminación con base en la religión. Quizá el caso que mejor ilustra el tono de las protestas haya sido el plantón en Shaheen Bagh, al norte de Delhi. Éste comenzó en diciembre de 2019 y se extendió hasta marzo de 2020, para protestar en contra de la enmienda y de la represión policiaca a estudiantes de la Universidad Jamia Millia Islamia, quienes se habían opuesto también. El plantón contó principalmente con la participación de mujeres musulmanas, pero fue escenario de una extraordinaria solidaridad intercomunitaria.

Las emotivas descripciones de Sushant Singh (2020) y Tavleen Singh (2020) sobre el plantón brindan un acercamiento a los sentimientos que unieron a los participantes. Sushant Singh narra cómo la llegada del nuevo año 2020 reunió en Shaheen Bagh a personas de distintas comunidades y clases sociales, quienes, al momento en que el reloj dio las 00:00, entonaron el himno nacional. Él destaca que los símbolos prominentes han sido la Constitución, la bandera y el himno nacional (S. Singh 2020). Por su parte, Tavleen Singh relata cómo para festejar el Día de la República (26 de enero) se hizo la lectura en voz alta 
del preámbulo de la Constitución. Era claro, dice ella, que los asistentes estaban allí para defender el texto y sus principios. En sus notas tomadas durante las conversaciones con las mujeres sobresale la convicción de que la Constitución y los derechos que contiene las protegerán (T. Singh 2020).

También se registraron protestas en Assam. No obstante, allí la comprensión de lo que la enmienda significa es totalmente opuesta: no se trata de un ataque contra el principio de no discriminación en la Constitución, sino de una estrategia del BJP para que pudieran salvarse aquellos extranjeros que hubieran quedado fuera de la actualización del Registro Nacional de Ciudadanos en Assam. Ante los resultados del NRC, es innegable que la enmienda podría utilizarse para salvar a muchos hindúes que quedaron fuera a pesar de que Narendra Modi declaró que la población de Assam no debía preocuparse al respecto (AntiCAB protests intensify in Assam, 10 things you need to know, 2019). El temor a volverse minoría en su propio estado permanece inalterable.

\section{CONSIDERACIONES FINALES}

Como señalé al principio, a primera vista la actualización en Assam del Registro Nacional de Ciudadanos y la Enmienda a la Ley de Ciudadanía (ahora aprobada) no parecen tener nada en común. De hecho, parecen funcionar con lógicas diferentes. Mientras el primero busca establecer quiénes son los extranjeros que de forma ilegal habitan en el noreste indio, para expulsarlos del territorio y de la nación india, el segundo se presenta como un mecanismo compasivo para agilizar el proceso de adquisición de ciudadanía de migrantes que hayan entrado como refugiados.

Sin embargo, como también quedó señalado, ambas decisiones tienen fuertes conexiones con concepciones de nación, ciudadanía, lengua, etnicidad y religión. Su origen está enraizado en políticas coloniales, pero también en la preocupación de India por mantener fronteras firmes hacia afuera (en particular con Pakistán y Bangladesh) y hacia dentro (sólo los ciudadanos deben estar presentes, los extranjeros no pueden encontrarse allí). Al mismo tiempo, como mostré, la creación de fronteras y Estados-nación en 1947 (India y Pakistán) y 
nuevamente en 1971 (Bangladesh) dividió poblaciones con fuertes lazos económicos, sociales, culturales y familiares.

La pandemia y el confinamiento en 2020 han significado una especie de pausa a esos dos mecanismos y sus efectos. ${ }^{6}$ Las consecuencias finales no estuvieron claras ni en 2019 ni en 2020. En declaraciones a la prensa, diversos funcionarios gubernamentales dieron la impresión de que la expulsión de los extranjeros ilegales era inminente, pero una pregunta emergió con claridad: ¿es acaso posible despojar de la ciudadanía a las personas? Y si así fuera, ¿adónde irían?, ¿a su supuesto país de origen? Bangladesh ha hecho declaraciones en el sentido de que no tiene intención de recibir a esas personas consideradas "extranjeras" (Assam NRC. What Next for 1.9 million stateless Indians? 2019).

La incertidumbre se cierne no sólo sobre estos sectores, sino sobre toda la población india en general. En 2003 se había introducido otra enmienda a la Ley de Ciudadanía. Ésta estipulaba, entre otros elementos, que era obligatorio el registro de los ciudadanos y la emisión de una tarjeta de identificación nacional para todos. Cuando en 2010 se introdujo la noción del Registro Nacional de Población (NRP, por sus siglas en inglés), se pensó que contendría los nombres de todos los residentes en India, incluyendo los extranjeros. Además de esto, habría algunos datos como nacionalidad, domicilio, nombre del padre y de la madre, lugar de nacimiento y ocupación, entre otros. Esta lista se preparó junto con la de hogares del Censo de 2011 y se actualizó en 2015. El Registro Nacional de Población se actualizaría antes del Censo de 2021 y sería la base para el Registro Nacional de Ciudadanos a nivel nacional (con excepción de Assam obviamente, pues ya se habría actualizado en ese estado) (Government of India 2010). Originalmente, la información habría sido complementada con la elaboración de la lista de hogares del Censo 2021.

El periódico The Hindu presentó una solicitud de información ante la Oficina del Registro General para conocer el estatus de este Registro Nacional de Población y en noviembre de 2020 se le respondió que se estaba finalizando el calendario o el cuestionario que acompañaría su actualización (V. Singh 2020). Sin embargo, su inicio, programado para abril de 2020, se pospuso debido a la pandemia.

\footnotetext{
${ }^{6}$ Como veremos más adelante, la pandemia ha puesto en duda que para 2021 se pueda actualizar en toda India el Registro Nacional de Ciudadanos.
} 
Cabe destacar que hay varios elementos preocupantes en esta actualización del NRP, a la que ya 13 estados y territorios de la Unión se han opuesto por su vinculación con la enmienda y el NRC en Assam. También en esta versión se preguntará a las personas la fecha y el lugar de nacimiento de su madre y de su padre, así como su último domicilio. La amenaza que se asoma detrás de estas preguntas (pues podrían arrojar información sobre una posible llegada a India sin documentación) ha hecho que gobiernos estatales como el de Bengala occidental y Rajasthan se opongan a ellas (V. Singh 2020).

La reproducción a nivel nacional de lo ocurrido en Assam (las dificultades experimentadas por la población debido a la falta de documentos, los problemas de literacidad y de inequidad de género y un número de personas fuera de la nación) está detrás de las críticas y la oposición al NRP. Pero, sobre todo, está la premisa (amenazante) de la que se parte: la capacidad de distinguir "fácilmente" entre el ciudadano y el extranjero, entre el residente legal y el ilegal.

Por todo ello se vuelve más urgente y preocupante entender estos dos mecanismos estatales, NRC y enmienda (ahora Ley de Ciudadanía 2019), pues vulneran la posición de la población, en vez de fortalecer la seguridad humana en India. A la luz de sus futuras ramificaciones, la idea de que la población india pueda vivir sin miedo y sin carencias y con dignidad parece alejarse, parafraseando la definición condensada de seguridad humana (United Nations Fund Trust for Human Security).

Laura Carballido Coria es profesora-investigadora del Departamento de Ciencias Sociales y Coordinadora del Posgrado en Ciencias Sociales y Humanidades, de la Universidad Autónoma Metropolitana, Unidad Cuajimalpa. Es licenciada en historia por la Universidad Nacional Autónoma de México y maestra y doctora en Estudios de Asia y África, área: India, por El Colegio de México. Sus líneas de investigación, desde la perspectiva de la historia social y urbana, incluyen los procesos históricos de la Partición de India (1947), el nacionalismo hindú, los proyectos de salud pública en Delhi colonial y las comisiones de investigación (inquiry commissions). 


\section{REFERENCIAS}

Ahmad, Nafis. 1947. The Basis of Pakistan. Calcuta: Thacker, Spink and Co.

Alagappa, Muthiah, ed. 1998. Asian Security Practice. Material and Ideational Influences. Stanford, California: Stanford University Press.

"Anti-CAB protests intensify in Assam, 10 things you need to know." 2019. The Indian Express, diciembre 12, 2019. https://indianexpress.com/article/north-east-india/ assam-burns-in-anti-cab-protests-ten-things-you-need-to-know-6163673/

“Assam NRC. What Next for 1.9 million stateless Indians?” 2019. BBC News, agosto 31, 2019. https://www.bbc.com/news/world-asia-india-49520593

Bagchi, Jasodahra y Subhoranjan Dasgupta, eds. 2006. The Trauma and the Triumph. Gender and Partition in Eastern India. Calcuta: Stree.

Barnett, Jon y W. Neil. Adger 2007. "Climate Change, Human Security and Conflict." Political Geography 26 (6): 639-655. https://doi:10.1016/j.polgeo.2007.03.003

Baruah, Sanjib. 2001. India Against Itself. Assam and the Politics of Nationality. Nueva Delhi: Oxford University Press.

Bashir Uddin, Mohammed. 2014. "Human Trafficking in South Asia: Issues of Corruption and Human Security." International Journal of Social Work and Human Services Practice 2 (1): 18-27. https://doi: 10.13189/ijrh.2014.020103

BJP. 2014. "Election Manifesto 2014." The Hindu. https://www.thehindu.com/ multimedia/archive/01830/BJP_election_manif_1830927a.pdf

BJP. 2019. "Election Manifesto 2019." The Times of India. https://timesofindia. indiatimes.com/realtime/BJP_Election_2019_english.pdf

Brass, Paul. R. 2004. The Politics of India since Independence. The New Cambridge History of India. Cambridge: Cambridge University Press.

Butalia, Urvashi. 1998. The Other Side of Silence. Voices from the Partition of India. Nueva Delhi: Viking. 
Carballido Coria, Laura. 2011. ¿India o Pakistán? Espacios divididos. México: El Colegio de México/Universidad Autónoma Metropolitana, Unidad Cuajimalpa.

Chandra, Bipan, Mridula Mukherjee y Aditya Mukherjee. 2002. India after Independence: 1947-2000. Nueva Delhi: Penguin Books.

Chatterjee, S. P. 1947. The Partition of Bengal. A Geographical Study with Maps and Diagrams. Calcuta: Calcutta Geographical Society.

Chatterjee Shibashis. 2014. "The Political Economy of Human Security in South Asia." En South Asia in Transition, editado por Chakma, Bhumitra, 195-229. Londres: Palgrave Macmillan. https://doi.org/10.1057/9781137356642_10

Chatterji, Joya. 1996. Bengal Divided. Hindu Communalism and Partition 1932-1947. Nueva Delhi: Cambridge University Press.

Commission on Human Security, United Nations. 2003. Human Security Now. Nueva York. https://reliefweb.int/report/world/human-security-now-protecting-and-empoweringpeople

Government of Assam. 2014a. "Filling up application forms." Office of the State Coordinator of National Registration (NRC), Assam. http://www.nrcassam.nic.in/receiptapplication-form.html

Government of Assam. 2014b. "Verification." Office of the State Coordinator of National Registration (NRC), Assam. http://www.nrcassam.nic.in/field-verification.html

Government of Assam. 2014c. "Timelines Chart." Office of the State Coordinator of National Registration (NRC), Assam. http://www.nrcassam.nic.in/timelines-chart. $\underline{\text { html }}$

Government of Assam. 2014d. "Training Materials for Resource Persons to Conduct Grama Sabha/Group Discussions at Community Level." Office of the State Coordinator of National Registration (NRC), Assam. http://nrcassam.nic.in/images/pdf/flip english . pdf 
Government of India. 1955. “The Citizenship Act, 1955.” https://www.indiacode.nic. in/bitstream/123456789/1522/1/a1955-57.pdf

Government of India. 2010. "Introduction to NPR 2010." Office of the Registrar General \& Census Commissioner, India. https://censusindia.gov.in/2011-common/ introductiontonpr.html

Government of India. 2019. "Citizenship Amendment Act." The Gazette of India. http://egazette.nic.in/WriteReadData/2019/214646.pdf

Hansen, Thomas B. 1999. The saffron wave. Democracy and Hindu nationalism in modern India. Nueva Jersey: Princeton University Press.

Hansen, Thomas B. 2000. "Governance and Myths of State in Mumbai." En The Everyday State and Society in Modern India, editado por C. J. Fuller y Véronique Bénéï. Nueva Delhi: Social Science Press.

Hazarika, Sanjay. 1995. Strangers of the Mist. Tales of War and Peace from India's Northeast. Nueva Delhi: Penguin Books.

Hazarika, Sanjoy. 2000. Rites of Passage. Border Crossings, Imagined Homelands, India's East and Bangladesh. Nueva Delhi: Penguin Books.

“How Fate of 19 lakh people hang hangs in balance in Assam after NRC's final list.” 2019. India Today, septiembre 3, 2019. https:/www.indiatoday.in/programme/in-depth/ video/in-depth-how-fate-of-19-lakh-people-hangs-in-balance-in-assam-after-nrc-sfinal-list-1595094-2019-09-03

“India and Bangladesh. Migration Claims Fact-Checked.” 2020. BBC News, febrero 21, 2020. https://www.bbc.com/news/world-asia-india-51575565

India Code. Digital Repository of All Central and State Acts: https://www.indiacode.nic.in/ “India excludes nearly 2 million from Assam citizen list.” 2019. Al Jazeera, agosto 31, 2019. https:/www.aljazeera.com/news/2019/08/31/india-excludes-nearly-2-millionpeople-from-assam-citizen-list/ 
“India probe after 'cow vigilantes kill Muslim man'." 2017. BBC News, abril 5, 2017. https://www.bbc.com/news/world-asia-india-39499845

Jaffrelot, Chistophe. 1996. The Hindu nationalist movement in India. Nueva York: Columbia University Press.

Jaffrelot, Christophe. 2015. "Narendra Modi and the power of television in Gujarat." Television and New Media 16 (4): 346-353. https://doi.org/10.1177/152747641557 5499

Jaffrelot, Christophe. 2017. "India in 2016. Assessing Modi Mid-Term.” Asian Survey 57 (1): 21-32. https://doi.org/10.1525/as.2017.57.1.21

Jalal, Ayesha. 1994. The Sole Spokesman. Jinnah, the Muslim League and the Demand for Pakistan. Cambridge: Cambridge University Press.

Jalal, Ayesha. 2001. Self and Sovereignty. Individuals and Community in South Asian Islam Since 1850. Nueva Delhi: Oxford University Press.

Khilnani, Sunil. 2004 [1997]. The Idea of India. Nueva Delhi: Penguin Books.

Martínez Saavedra, Beatriz. 2016. "Comicios 2014 y la victoria de Narendra Modi. ¿Desarrollo económico o polarización social?” Foro Internacional 2: 450-469. https://doi.org/10.24201/fi.v56i2.2321

Mehta, Pratap Bhanu. 2019a. "A hundred days on, Modi 2.0: Its purpose is the show of power, nationalist fervour, social control.” The Indian Express, septiembre 10, 2019. https://indianexpress.com/article/opinion/columns/narendra-modi-bjp-hundreddays-kashmir-lockdown-5980799/

Mehta, Pratap Bhanu. 2019b. "Staggering dominance: The only authentic analysis of this election is two words - Narendra Modi." The Indian Express, mayo 24, 2019. https://indianexpress.com/article/opinion/columns/narendra-modi-lok-sabhaelections-2019-results-bjp-congress-rahul-gandhi-5745371/

Menon, Ritu y Bhasin Kamla. 2000. Borders and Boundaries. Women in India's Partition. Nueva Delhi: Kali for Women. 
Middleton, Townsend. 2015. "La antropo-lógica. Paradigmas de la identidad moderna". En Otras modernidades: historias, culturas, identidades, editado pot Saurabh Dube y Banerjee Ishita, 285-304. México: El Colegio de México.

“Newton' Modi has a lot to answer." 2002. The Times of India, marzo 2, 2002. https://timesofindia.indiatimes.com/india/Newton-Modi-has-a-lot-to-answer/ articleshow/2599766.cms

“NRC An Experiment Gone Awry." 2019. The Hindustan Times, septiembre 15, 2019. https://www.hindustantimes.com/editorials/nrc-an-experiment-gone-awry/storyUts6kjelYErFYeRmGWBuKM.html

Organización de las Naciones Unidas. s. f. “¿Qué es la Seguridad Humana?” https://www. un.org/humansecurity/es/what-is-human-security/

Organización de las Naciones Unidas. 2012. "Migrants by Origin and Destination. The Role of South-South Migration.” Population Facts 2012, núm. 3 (junio). https://www. un.org/en/development/desa/population/publications/pdf/popfacts/popfacts_20123 _South-South_migration.pdf

Samaddar, Ranabir, ed. 1997. Reflections on Partition in the East. Nueva Delhi: Vikas.

Savarkar, Vinayak D. 1969 [1923]. Hindutva. Who is a Hindu? Bombay: Veer Savarkar Prakashan.

Singh, Sushant. 2020. "With a flag, a song and book. Reclaiming national symbols is an act of political genius and imagination." The Indian Express, enero 24, 2020. https://indianexpress.com/article/opinion/columns/shaheen-bagh-caa-protests-nrcmuslims-national-anthem-with-a-flag-song-and-book-6232185/

Singh, Tavleen. 2020. "Celebrate the Constitution in these troubled times." The Indian Express, enero 26, 2020. https://indianexpress.com/article/opinion/columns/shaheenbagh-protests-caa-nrc-development-army-constitution-pakistan-celebrate-theconstitution-6235413/

Singh, Vijaita. 2020. "National Population Register Questionnaire is Being Finalised, Says Registrar General of India." The Hindu, noviembre 18, 2020. https://www.thehindu. 
com/news/national/national-population-register-questionnaire-is-being-finalisedsays-registrar-general-of-india/article33126299.ece

Talbot, Ian. 1998. Pakistan. A Modern History. Nueva York: St. Martin's Press.

Thapar, Romila. 1989. "Imagined Religious Communities? Ancient History and the Modern Search for a Hindu Identity." Modern Asian Studies (23): 209-231.

United Nations Trust Fund for Human Security. s. f. "Human security Milestones and History". https://www.un.org/humansecurity/human-security-milestones-and-history/

Unesco. 2013. "India: Sahajani Shiksha Kendra: Alfabetización y educación para el empoderamiento de las mujeres." Programas de alfabetización centrados en las mujeres para reducir las desigualdades de género, 48-51. Hamburgo: Unesco. https://unesdoc.unesco.org/ark:/48223/pf0000231720

Van der Veer, Peter. 1994. Religious Nationalism. Hindus and Muslims in India. Berkeley: University of California Press.

Yousaf, Farhan N. 2017. "Human (In)Security in South Asia." South Asian Studies. A Research Journal of South Asian Studies 32 (2): 481-497. 